\title{
Deep learning-based forecasting of aggregated CSP production
}

\author{
Jorge Segarra-Tamarit, Emilio Pérez*, Eric Moya, Pablo Ayuso, Hector Beltran \\ Department of Industrial Systems Engineering and Design, Universitat Jaume I, Castelló de la Plana, Spain.
}

\begin{abstract}
This paper introduces deep learning-based forecasting models for the continuous prediction of the aggregated production generated by CSP plants in Spain. These models use as inputs the expected top of atmosphere irradiance values and available weather conditions forecasts for the locations where the main CSP power plants are installed. The performances of the forecast models are analysed and compared by means of the most extended metrics in the literature for a whole year of CSP energy production.
\end{abstract}

Keywords: Concentrated solar power, deep learning, neural networks, forecasting.

\section{Introduction}

Concentrated solar power (CSP) has experienced a very significant growth in Spain in the last decade, going from an aggregated generation of $130 \mathrm{GWh}$ in 2009 to $4,424 \mathrm{GWh}$ in 2017 [1], this accounts for the $1.5 \%$ of the total electricity production and contributes with 1,464 M€ to the spanish GDP. After all this evolution, there are nowadays 2,300 MW of installed power [2], so that Spain encounters almost half of the world's installed capacity.

CSP systems mainly generate power by concentrating direct normal irradiance (DNI) by means of mirrors or lenses and heating up a fluid that drives a turbine connected to a power generator [3]. Nevertheless, the stochastic cloud movement patterns throughout the day prevent these plants from granting a predictable production at any given moment, which hinders the participation of this technology in the electricity markets of the different countries. Typically, this drawback has been overcome by integrating CSP plants with storage capacity in the form of thermal energy in molten salts, which is released for generating electricity whenever it is needed or more profitable [4], whether day or night.

CSP forecasting is a relatively recent topic of research, since its worldwide deployment has been significantly lower than that of other renewable energy sources, such as wind or solar photovoltaic power plants. Indeed, the main efforts in this direction, and differently to those for solar photovoltaic forecasting, have been made in order to forecast the DNI since it is the fundamental input for this type of energy production, as diffuse radiation cannot be effectively concentrated [5, 6]. Furthermore, literature related to DNI forecasting mainly focuses on single location installations, being the goal of the predictions to optimize the charge and discharge storage system schedule and the plant operation [7, 8]. A good review of the different DNI forecasting techniques can be found in [6], where the authors compare numerical weather prediction (NWP) models, time series analysis methods, cloud motion vectors and hybrid methods.

Neural networks (NN), which have been recently granted a huge amount of attention in the context of time series prediction [9] and energy systems [10], have also been proposed for this field. For instance, [11] proposes a global solar irradiance prediction and its application to CSP production planning. Authors in [12] also use these techniques to obtain the different components of radiation in a single location. Convolutional neural networks, long short-term memory networks, deep belief networks, and autoencoders are also used in $[13,14]$ to forecast the photovoltaic power for individual power plants.

On the other hand, there are studies centered in aggregated solar production at regional or country levels, but their focus is usually related to long-term analysis. In these cases, the forecasting is usually carried out by taking long-term

*pereze@uji.es 
past data (years) and assuming that the conditions will be similar in the future. Examples of this kind of studies are [15] and [16].

In opposition to all these previous proposals, the goal of this paper is to develop a short-term deep learningbased forecasting model that continuously predicts the aggregated CSP energy production in Spain, and even that provided by the thermal storage units during night hours. Because of the already introduced significant growth of this technology in the country's energy mix in the last years, a good forecast of this production becomes increasingly important for the transmission system operator to manage the network. Furthermore, because of the marginal prices nature of the iberian electricity market (MIBEL market that groups Spain and Portugal), the aggregated CSP energy production can affect the final electricity prices during some hours along the year. Therefore, a forecast can be used as an input for price models used by market agents. Note however that the aggregated forecast should also include the effect of the behaviour of the different plant operators and when they are using their storage capabilities. In this sense, this is a completely different (and harder to solve) problem than the forecast of the aggregated instantaneous DNI received by the plants. To the authors' knowledge, there are no works in the literature specifically devoted to this problem, while our proposal also takes it into account.

The structure of the paper is as follows. In the next section, the different data sources for the models are described. Then, in section 3, the performance metrics used to evaluate the models are presented. Section 4 proposes a simple naive model, which allows the comparison for the neural network-based models in sections 5 and 6 . In section 7, the results for each model are presented and discussed. Finally, some conclusions are introduced in section 8.

\section{Data sources for the models}

In the following sections, different forecasting models will be proposed for the short-term prediction of the aggregated CSP production in Spain. Since CSP installations are gathered in a limited number of locations, mainly in the southern part of the country to take advantage of a higher solar resource availability, the amount of energy they produce depends mostly on the solar irradiance arriving to the surface in those locations and on the peak power of the plants.

Therefore, the proposed forecasting models use as inputs four types of data: an estimation of future irradiance at the top of atmosphere (TOA) for those locations, which can be precisely determined as a function of the geographic coordinates, date, and time; a cloud cover forecast, which affects the quantity of that irradiance that arrives to the surface; the hour of the day, because the stored energy is delivered only during the nighttime; and the external temperature, as the energy is stored with molten salts [17]. For some models, also day of the year encoded as a value that is 0 for December the 21 st and increases linearly until June the $21 \mathrm{st}$, is used, so that the model is allowed to consider seasons of the year.

For this work, data corresponding to the year 2016 in Spain are downloaded from different sources to provide the inputs for the models. Furthermore, the actual power generation along that year is also needed to train the neural network-based forecasts and for validation purposes.

\subsection{Locations and peak powers}

Location and peak power of the CSP power plants in Spain are obtained from the Spanish association for promoting the CSP industry, Protermosolar [1].

In Spain, there are 50 power plants with a total peak power of $2.3 \mathrm{GW}$. As previously discussed, the majority of these power plants are built in the southern regions of the country and some of the installations are close to each other. Hence, some of the nearby facilities are considered as a single one with the aggregated peak power, leading after that to a number of 25 considered locations, with the total power remaining unchanged. Rated peak power, coordinates and location for these groups are shown in Table 1.

\subsection{Top-of-atmosphere solar irradiance forecast}

The solar irradiance in a horizontal plane on the top of the atmosphere, $I_{\mathrm{TOA}}$, depends on the hour angle in the local solar time $(h)$, the current declination of the Sun $(\delta)$, and the local latitude $(\Phi)$. These factors can be taken into account with a single parameter, the solar zenith angle $\left(\theta_{s}\right)$, according to the expression:

$$
\cos \theta_{s}=\sin \Phi \sin \delta+\cos \Phi \cos \delta \cos h
$$


Table 1: Location and power (MW) of the considered CSP groups

\begin{tabular}{lccc}
\hline Location & Latitude & Longitude & Power \\
\hline Logrosán & 39.34 & -5.49 & 200 \\
Alcázar de San Juan & 39.39 & -3.21 & 200 \\
Sanlúcar la Mayor & 37.39 & -6.20 & 180 \\
Aldeire & 37.16 & -3.07 & 150 \\
Palma del Rio & 37.70 & -5.28 & 150 \\
Badajoz & 38.88 & -6.97 & 150 \\
Torre de Miguel Sesmero & 38.62 & -6.79 & 150 \\
San José del Valle & 36.61 & -5.79 & 100 \\
Morón de la Frontera & 37.12 & -5.45 & 100 \\
Écija & 37.54 & -5.08 & 100 \\
El Carpio & 37.94 & -4.49 & 100 \\
Navalvillar de Pela & 39.09 & -5.47 & 100 \\
Arenas de San Juan & 39.22 & -3.50 & 100 \\
Lebrija & 36.92 & -6.01 & 50 \\
Fuente Palmera & 37.70 & -5.10 & 50 \\
Villena & 38.64 & -0.87 & 50 \\
Olivenza & 38.68 & -7.10 & 50 \\
Puertollano & 38.69 & -4.11 & 50 \\
Orellana & 39.01 & -5.53 & 50 \\
Talarrubias & 39.03 & -5.23 & 50 \\
Majadas & 39.94 & -5.75 & 50 \\
Azaila & 41.29 & -0.49 & 50 \\
Calasparra & 38.23 & -1.70 & 31 \\
Lleida & 41.50 & 0.87 & 24 \\
Fuentes de Andalucía & 37.46 & -5.35 & 20 \\
\hline
\end{tabular}

Therefore, $I_{\text {TOA }}$ can be precisely determined from $\theta_{s}$.

\subsection{European Centre for Medium-Range Weather Forecasts}

Weather forecasts are obtained from the European Centre for Medium-Range Weather Forecasts (ECMWF) [18]. The Centre produces an ensemble of predictions that indicate the likelihood of a range of future weather scenarios. Forecasts are made using NWP methods, which have good performances for day-ahead forecasts and beyond. The forecasts predict the next 10 days with 1-hour time steps, and updated forecasts are available every 12 hours. These forecasts are frequently used in the literature to quantify the impact of forecasted variables on the operation of a CSP plant [19] or on its annual incomes [20].

In this work, the 12 hours comprehended between one forecast and the new one are used. The spatial resolution of the forecasts is $0.1^{\circ}$ for both latitude and longitude, which allows to obtain 25 forecasts time series corresponding to the CSP groups in Table 1. As a first approach to the problem, only temperature and total cloud cover $(\widehat{\mathrm{cc}})$ forecasts are used. The latter is calculated from the cloud occurring at different model levels through the atmosphere, and represents the proportion of a grid box covered by clouds. This parameter varies from 1, for a totally covered sky, to 0 , for a clear sky situation. The main limitation for $\widehat{c c}$ is that it does not consider the clouds transmittance. This can cause that, for two situations with the same $\widehat{\mathrm{cc}}$, the forecasted CSP generation can be different if the clouds have different transmittances.

\subsection{Measured power generation}

The hourly CSP generation for each province in the whole country is obtained from ESIOS, the information system of Red Eléctrica de España, Spain's TSO [21]. This service provides all the public information related to the Spanish 
electricity system from the point of view of the operation. More specifically, it provides information about forecasting, scheduling, measurements, settlements and demand management, among others. This service provides an API which has been used to obtain the data.

In this work, the amount of CSP-based power generated hourly by each province in 2016 is downloaded and then aggregated to obtain the total for the country. These datasets are not inputs for the different models, but they will be used for training and validation purposes. Different CSP production statistics during 2016 are shown in Table 2. It should be noted that the CSP generation during hot months accounts for a $77 \%$ of the yearly generation.

\begin{tabular}{lcccc}
\multicolumn{5}{l}{ Table 2: CSP production statistics during $2016(\mathrm{GW})$} \\
\hline Season & Mean & min & max & std \\
\hline All & 0.577 & 0 & 2.22 & 0.717 \\
Summer & 0.887 & 0 & 2.22 & 0.778 \\
Winter & 0.267 & 0 & 2.17 & 0.483 \\
\hline
\end{tabular}

\section{Performance metrics}

Different performance metrics are used for the comparison of forecasting models in the literature. In this work, several of these metrics will be used in order to analyze a wide variety of aspects. All these errors will be obtained by comparing the real measured value for each time step $\left(y_{k}\right)$ with the forecast value $\left(\hat{y}_{k}\right)$.

The first performance metric is the mean absolute percent error (MAPE), i.e. the mean of the absolute values of the ratio of the errors to the actual observed values.

$$
\text { MAPE }=\frac{100}{N} \sum_{k=1}^{N}\left|\frac{\hat{y}_{k}-y_{k}}{y_{k}}\right|
$$

MAPE is proposed because it is one of the most common metrics of a forecast accuracy. However, it has several problems [22]. Notice from (2) that MAPE becomes infinite when $y_{k}$ is 0 and, furthermore, this error becomes very large for small $y_{k}$ values. For this reason, choosing forecasts with low MAPE tends to systematically reduce error for lower values of $y_{k}$ and, therefore, favors lower predictions.

In the field of solar energy forecasting, it is a common practice for the sake of a better measure of errors to only calculate the performance during sunny hours, with a solar zenith angle criteria [23]. However, for the forecasting of power produced by CSP with molten salt storage, a different approach must be taken due to the existence of accumulated energy that is released during nights. In this sense, MAPE is computed for any time step for which $y_{k}>0$ whereas the rest of the performance metrics are computed for each time step.

In this sense, because of the limitations of MAPE, other metrics are proposed to be considered along with it. The second one is the mean absolute error (MAE).

$$
\text { MAE }=\frac{1}{N} \sum_{k=1}^{N}\left|\hat{y}_{k}-y_{k}\right|
$$

As the MAE does not consider if the forecast error is upwards or downwards, the bias (4) is used to assess such aspect. A negative bias will represent an underestimation, i.e. the model being lower than the real values, while a positive bias will represent an overestimation.

$$
\text { Bias }=\frac{1}{N} \sum_{k=1}^{N}\left(\hat{y}_{k}-y_{k}\right)
$$

Moreover, root mean square error (RMSE) is also used to evaluate the average spread of the forecasting errors.

$$
\mathrm{RMSE}=\sqrt{\frac{1}{N} \sum_{k=1}^{N}\left(\hat{y}_{k}-y_{k}\right)^{2}}
$$


MAE and RMSE, being absolute metrics, show difficulties when trying to compare different time series. However, in this work they will only be used for the comparison of different forecasting models on the same series.

The Pearson correlation coefficient, $r$, will be also used as performance metric, representing the linear correlation between the measured data and the forecasts obtained from the different models. This coefficient is obtained following the equation

$$
\mathrm{r}_{\hat{y} \mathrm{y}}=\frac{\operatorname{cov}(\hat{Y}, Y)}{\sigma_{\hat{Y}} \sigma_{Y}},
$$

where $\hat{Y}$ and $Y$ are $N$-length arrays of the forecast and real measured values, $\sigma_{\hat{Y}}, \sigma_{Y}$ are the standard deviations of these arrays and cov is the covariance between both time series.

Finally, the performance of every model can be compared with a reference one using skill metric (7). In this case, the naive model, described in the next section, will be used as a reference.

$$
\text { Skill }=\left(1-\frac{\text { RMSE }_{\text {Forecast }}}{\text { RMSE }_{\text {Naive }}}\right) 100
$$

Notice that, for a forecast with exactly the same RMSE as the naive model, Skill equals 0. If RMSE is lower than the one for the naive model, then Skill is positive and, if it is higher, Skill is negative. A perfect forecast $(\mathrm{RMSE}=0)$ would give skill of 100 .

\section{Naive approach}

At first, a naive approach for the forecast of the aggregated CSP production is proposed. This model makes use of the cloud cover forecast $(\widehat{\mathrm{cc}})$ to obtain the ratio of passing irradiance as $(1-\widehat{\mathrm{cc}})$ which is then multiplied by $I_{\mathrm{TOA}}$, in order to calculate an estimation of the irradiance on the surface for each of the 25 CSP locations. Finally, these estimated irradiances are multiplied by the peak power of each CSP installation $\left(\right.$ Ppeak $\left._{i}\right)$ and they are all added up to obtain the naive estimation of aggregated power generation, $P_{N}(8)$. As Ppeak is given for standard conditions, $I_{\mathrm{TOA}}$ is divided by $1000 \mathrm{~W} / \mathrm{m}^{2}$.

$$
P_{N}=\sum_{i=1}^{25} \frac{I_{\mathrm{TOA}_{i}}}{1000\left[W / m^{2}\right]} \cdot \text { Ppeak }_{i} \cdot\left(1-\widehat{\mathrm{cc}}_{i}\right)
$$

Fig. 1 compares the real power measurements with the naive estimation for six different days (three consecutive summer days and three consecutive winter days). Dots in the solid line represent the time instants for which ECMWF model is calculated and, therefore, new cloud cover data are available.

It can be seen how this model performs fairly well during the daytime (although better in summer than in winter), but it clearly does not during the nighttime, when the forecast is always 0. Obviously, this is because this model lacks the ability to forecast the stored energy during the day, which is released at night. Note also how the tendency of the prediction changes right after the cited dots in the solid line, i.e. when new cloud cover forecasts become available.

Nevertheless, it is not straightforward to determine the contribution to the generation made by the molten salts storage during nights although, as the energy is stored in a thermal form, external temperature seems to be an important parameter to include in the model.

In order to quantify the influence in the CSP generation of the considered input variables, Table 3 shows the correlations between the CSP generation and the studied variables at different times of the day. It can be seen how, as studied in [24], the irradiance is the most important parameter (at least during daytime). Temperature and day of year also have a considerable impact in the generation whereas the hour of the day, at least encoded as a number between 0 and 24 , has the lower correlation. In the case of temperature, since it has a slower system dynamic than the irradiance, the correlation is maximum (with a value of 0.70) when comparing the current CSP generation with the temperature lagged by 2 hours .

This kind of problem, for which the relevant inputs are known but their particular effect is not, is suitable to be formulated as NNs in order to get more accurate forecasts. 


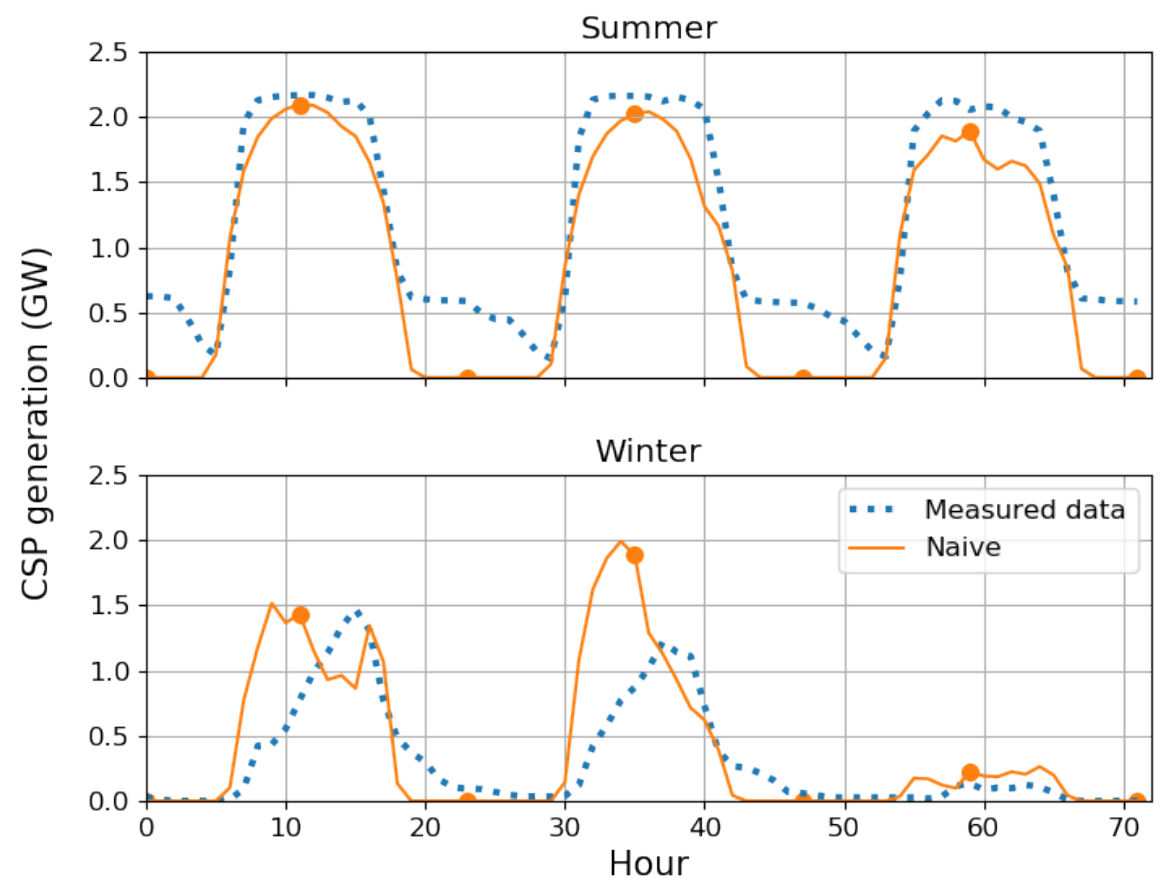

Figure 1: Naive model forecast for summer and winter days.

Table 3: Correlation coefficients between the CSP generation and the considered model inputs

\begin{tabular}{lcccc}
\hline Time of the day & $\mathbf{I}_{\mathrm{TOA}}\left(\mathbf{1}-\widehat{\mathrm{cc}}_{\mathbf{i}}\right)$ & Temperature & Hour & Day of year \\
\hline All & 0.88 & 0.66 & 0.09 & 0.48 \\
Daytime & 0.86 & 0.65 & 0.08 & 0.52 \\
Nights & -0.03 & 0.61 & 0.19 & 0.64 \\
\hline
\end{tabular}

\section{Feedforward neural network approach}

The field of artificial neural networks (ANN) and, more particularly, deep learning [25], is stirring up with great success many research fields. Some of their applications, such as audio or image recognition, are very well known, but some others, such as the search of new physical particles [26], are completely new fields. Although theory behind ANNs has been known for many years, it is not until the growth of computing power achieved with GPUs and the great amount of available information on many fields (big data), that they have started to be widely used in recent relevant problems.

Deep learning is a machine learning technique, based on ANN that allows to define a network architecture stacking several simple layers that apply non-linear operations to the network inputs. Before the practical use of the model, a training process is performed in which parameters of the different layers are learned from examples given to the ANN in a supervised learning process.

For the problem at hand, the forecasting of global CSP production, the main inputs considered are the 25 products of $I_{\mathrm{TOA}_{i}} \cdot$ Ppeak $_{i} \cdot\left(1-\widehat{\mathrm{cc}}_{i}\right)$, the 25 temperature forecasts and the hour of the day. Irradiance and temperature are used because they have the highest correlations in Table 3. However, the day of the year is not used because its information could be implicit in the temperature and irradiance values. The ANN proposed at this step presents three hidden layers with, respectively, 13, 3 and 3 neurons, rectified linear unit (ReLU) activations and a single output, $P_{N}$. In this work, all ANNs are programmed using Python 3.6 and Tensorflow 1.3 and trained to minimize the mean square error using 
the Adam optimizer with a 0.01 learning rate and neither regularization nor dropout. Moreover, $47 \%$ of the available data are used to train the models, $20 \%$ are used to perform the early stopping and validation of different model architectures and the final $33 \%$ are used for the testing of final results. Fig. 2 displays the forecasts obtained with this new model. In this case, it can be seen how the energy released during nights is being predicted. Table 4 compares, by means of its MAE, the performance of this feedforward NN (FF in the table) with the Naive model, considering separately winter and summertime. It can be seen how there is a big improvement for the summer forecasting, and a smaller one for winter.

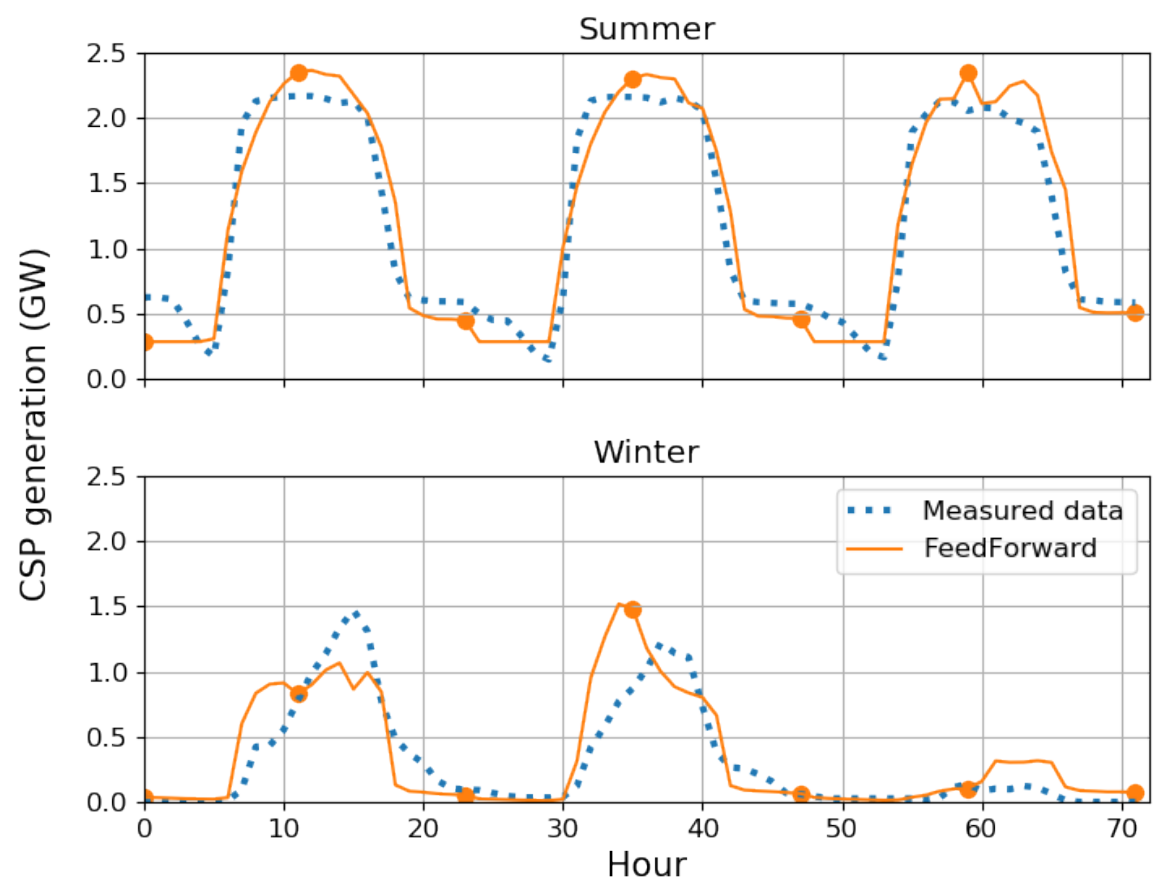

Figure 2: Feed forward model forecast for summer and winter days.

In order to further improve the forecast, and considering the correlations from Table 3, the day of the year is introduced as an additional input for a second model with the same ANN architecture, which can be seen in the right side of Fig. 4. The seasonal results for this improved model are also shown in Table 4 with the label FF+day. In this case, although the performance for summer is similar to the previous model, the results are significantly improved for winter days. Fig. 3 compares these two feedforward models during three consecutive winter days. It can be noticed how the addition of the day as a new input improves the results during nights significantly.

Table 4: MAE for winter and summer

\begin{tabular}{lcc}
\multicolumn{3}{c}{ Table 4: MAE for winter and summer } \\
\hline Model & MAE Summer & MAE Winter \\
\hline Naive & 0.32 & 0.16 \\
FF & 0.21 & 0.14 \\
FF + day & 0.20 & 0.11 \\
\hline
\end{tabular}




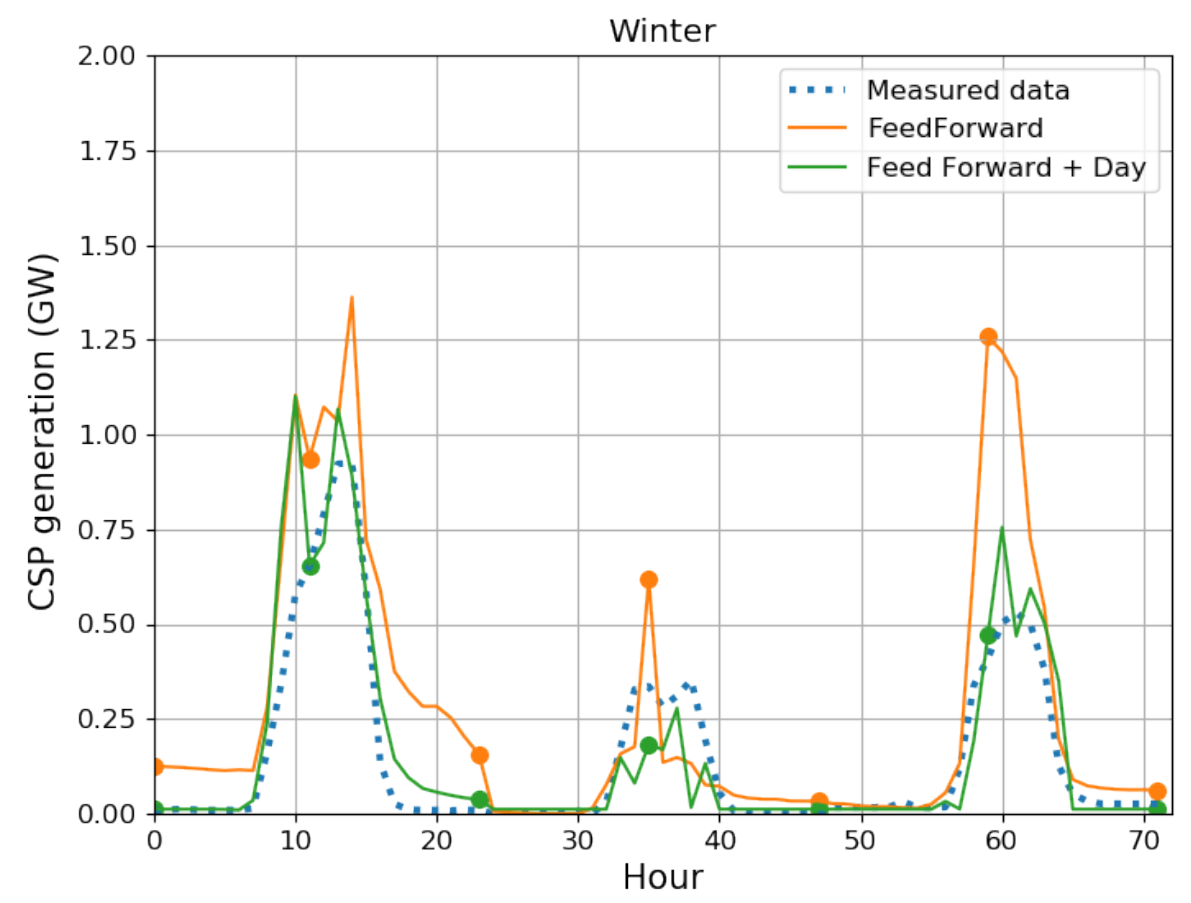

Figure 3: Comparison between two feedforward models with and without the day of the year as an input for three winter days.

\section{Recurrent neural network approach}

For the previous forecast models, both naive and ANN-based, $P_{N}$ is predicted by means of making different mathematical operations over simultaneous inputs. That is, at the moment the model is run, the prediction for each of the future 12 hours depends only on information for that same time instant $\left(I_{\mathrm{TOA}}\right.$ and $\left.\widehat{c c}\right)$. However, because of the way CSPs work, the generated power not only depends on the instantaneous inputs but also on the amount of energy that has been stored before by heating up the molten salts, hence on $I_{\mathrm{TOA}}$ and $\widehat{c c}$ on the previous periods.

This kind of behaviour can be more suitably addressed by means of recurrent neural networks (RNN), which are a different kind of ANN containing internal loops, allowing information to persist [27]. RNNs with traditional architecture show difficulties to track long-term dependencies, and thus have problems in the training stage [28]. Recently, a new kind of RNN, long short-term memory networks (LSTM) [29], has been proposed to solve these problems, improving the training speed and the ability to learn long-term dependencies.

In this work, a LSTM approach is finally proposed with the same inputs as the feedforward models, i.e. $I_{\mathrm{TOA}_{i}}$. Ppeak $_{i} \cdot\left(1-\widehat{c c}_{i}\right)$, temperature forecasts, time of day and day of the year, but the first two inputs are then provided as data series for all the prediction horizon instead of as single values. Therefore, $P_{N}$ forecasts for all the prediction horizon are also obtained. Whenever input data are updated, the model is run again.

In order to obtain the best possible LSTM architecture, a random search is run to select the best among a set of candidate values for a number of hyperparameters. The finally proposed network architecture presents a single LSTM layer, with an adjustable number of units and different possible types of activation, followed by an adjustable number of dense layers, which in turn also have different possibilities for activation and number of neurons. The range of all the considered values for the hyperparameters in the random search is summarized in Table 5 . The best performing models were obtained using a learning rate of $10^{-3}$, a regularization of also $10^{-3}$ and one dense layer between the LSTM and the output. Furthermore, most of the best solutions use "tanh" activation for the intermediate dense layer and ReLU activation for the LSTM and the output layer. The best performance is achieved by a number of combinations of hyperparameters. Among them, the architecture with the minimum number of parameters is chosen. 
This architecture features an LSTM layer with 30 hidden units and 24 time steps, followed by two fully connected layers of 43 and 24 neurons, respectively. The layers used in this model and the data dimensions are shown on the right side of Fig. 4.

Table 5: Random search hyperparameters

\begin{tabular}{lcc}
\hline Hyperparameter & Type & Search values \\
\hline Learning rate & Choice & $10^{-1}, 10^{-2}, 10^{-3}$ \\
L2 regularization & Choice & $10^{-1}, 10^{-2}, 10^{-3}$ \\
LSTM units & Continuous & 10 to 200 \\
LSTM activation & Choice & ReLU, tanh \\
Number of densely connected layers & Choice & $1,2,3$ \\
Dense neurons & Continuous & 24 to 200 \\
Dense activation & Choice & ReLU, tanh \\
Output activation & Choice & ReLU, tanh \\
\hline
\end{tabular}

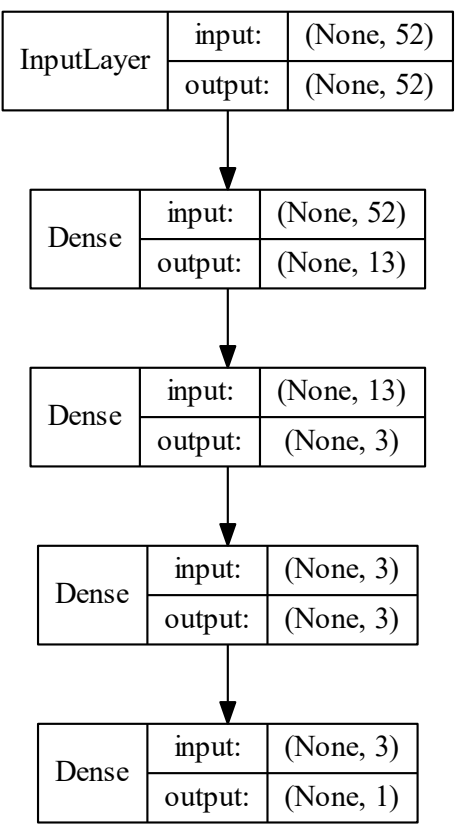

(a) FF+day

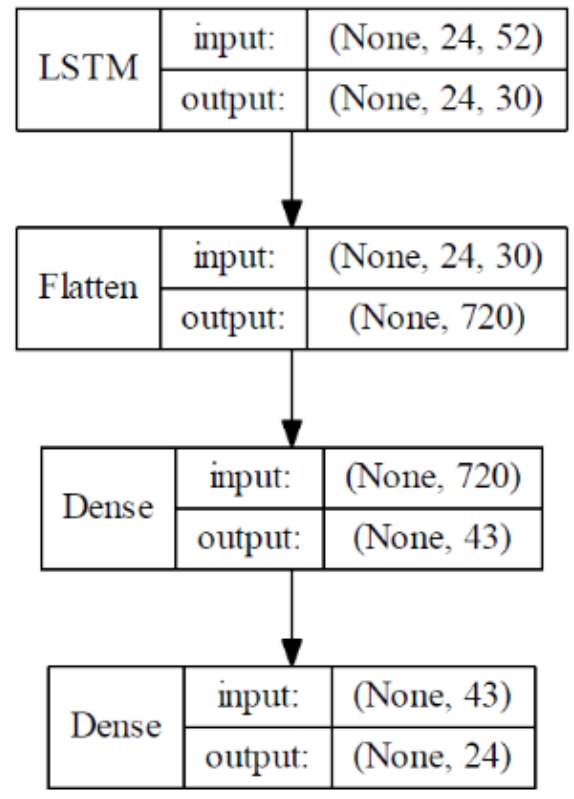

(b) LSTM

Figure 4: ANN architectures for the FF+day model (a) and the LSTM model (b)

Fig. 5 shows the forecasts provided by this model, showing a much more precise prediction of the accumulated power released during nights. 


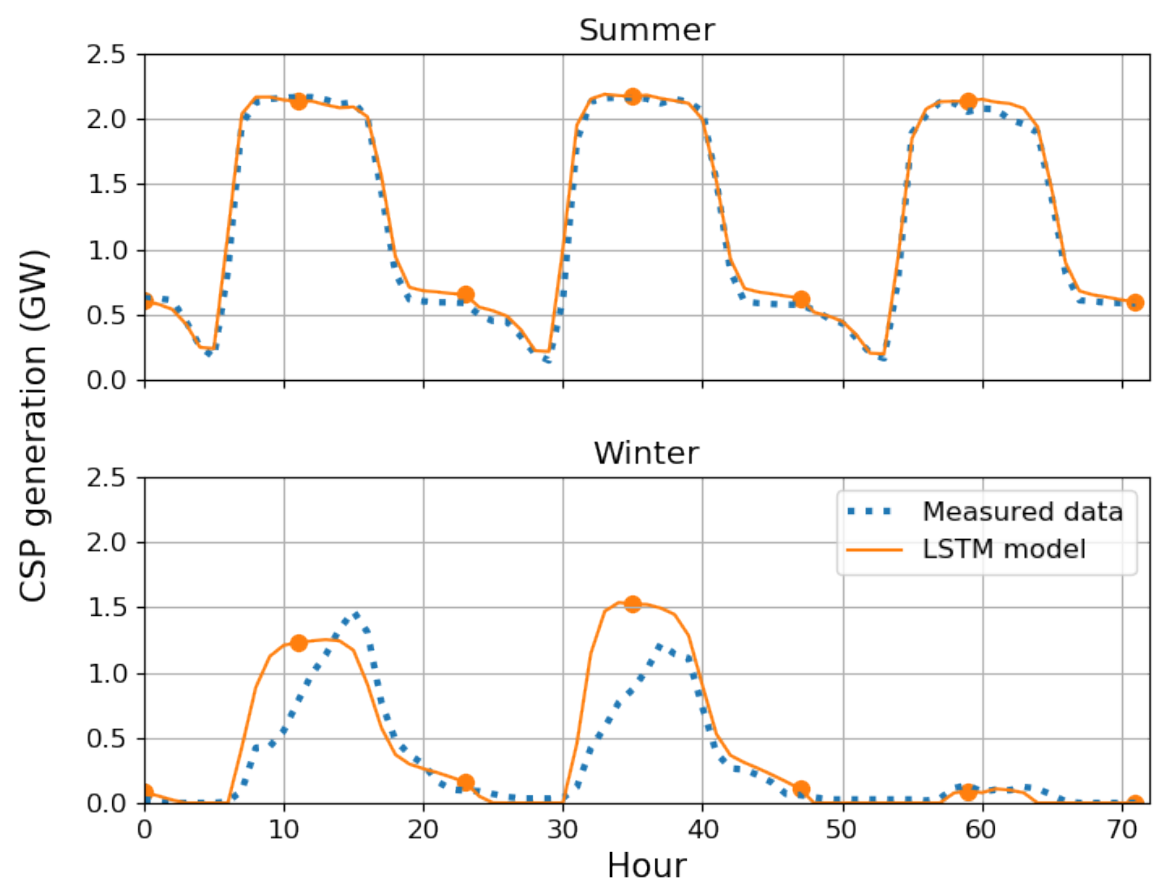

Figure 5: LSTM model forecast for summer and winter days.

\section{Results}

In this section, forecasting results for the RNN proposal will be compared against the naive and the FF approaches. However, one could argue that the LSTM model architecture chosen after the random search has a higher amount of trainable parameters and more information from previous time steps and, therefore, the direct comparison would be somewhat unfair. Therefore, a new feedforward neural network (FFbig), with the same inputs $(24 \times 52)$ and the same amount of trainable parameters $(42,019)$ as the LSTM, is trained. Furthermore, three other models are also developed for comparison purposes:

- A support vector regression (SVR) model implemented using sklearn with the same inputs as the LSTM, whose hyperparameters are also chosen by a random search algorithm. The obtained values are: epsilon $=0.115$, gamma $=0.01, \mathrm{C}=2.5$ and a radial basis function kernel.

- A model with Gated Recurrent Units (GRU) like the one proposed in [30], as these units are quite similar to LSTM ones, but just have one less gate. This GRU model features 20 units and two dense layers, with 109 and 24 neurons respectively, and is trained with the same inputs as the LSTM one. The resulting model has a few more parameters than the original LSTM.

- And finally, a new LSTM model using the direct normal irradiance ( $\widehat{\mathrm{DNI}})$ instead of $I_{\mathrm{TOA}}$ and $\widehat{c c}$ (LSTMDNI). Since the cloud cover variable only takes into account the percentage of the sky covered by clouds, and not their transmittance. Results might improve with such a model.

As a first approach to compare all the proposed models, Fig. 6 shows the measured data (x axis) and the forecast from each model (y axis) for the whole year 2016. The diagonal line would be a perfect forecast, while points below the line are the hours when a model underestimates the generation and points above the line are the opposite case. It can be seen how LSTM performance seems clearly superior to the naive and initial FF models, as most of the points are closer to the diagonal line. Initial FF models also seem to perform better than the naive approach, for which it 
can be seen a greater number of points with 0 prediction with respect to the other models. These points correspond to nights when, as discussed, the naive model is unable to predict generation. The proposed LSTM also seems to outperform the LSTMDNI, which features several points with an overestimation of the production. However, from the scatter plots it is difficult to tell if the LSTM performs better than FFbig, SVR and GRU.

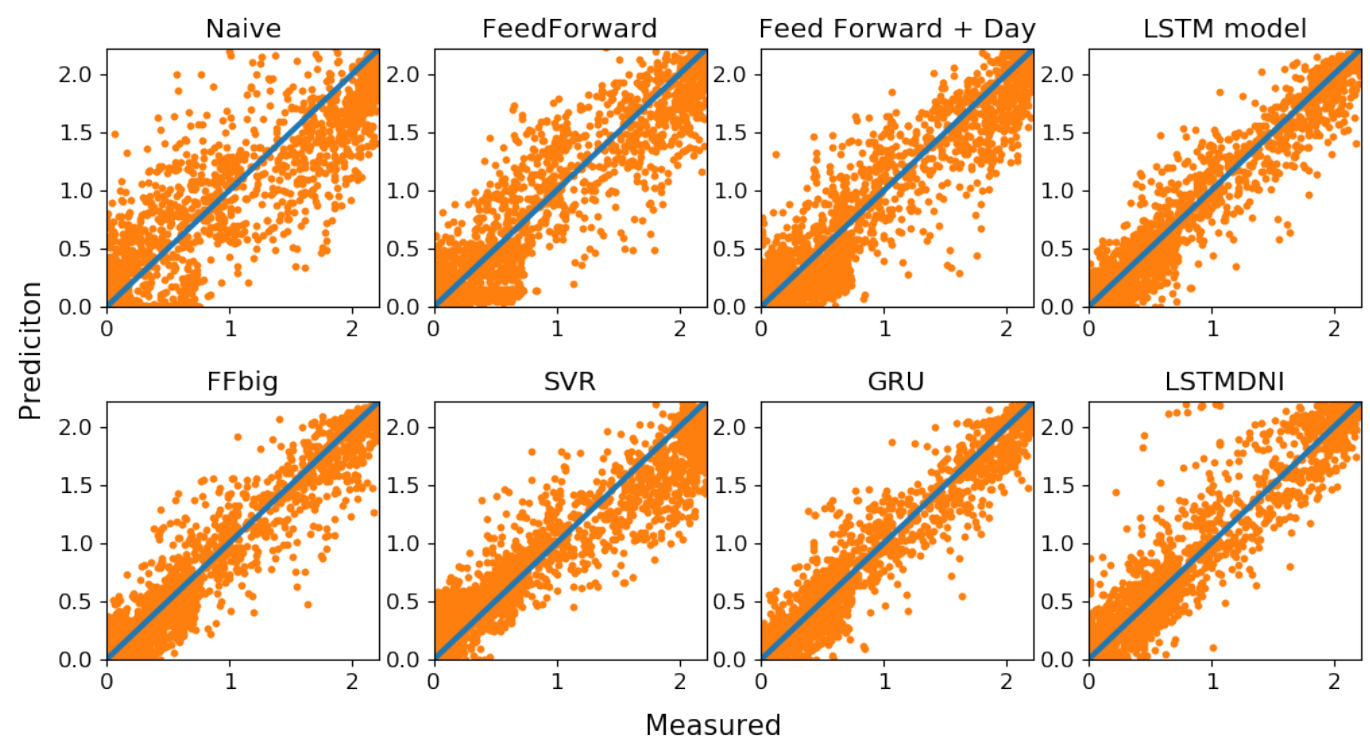

Figure 6: Performance of the eight models.

For a better comparison among models, and in addition to Fig. 6, the performance metrics described in section 3 are also computed for each proposed model for the testing data, considered both globally and separating summer (April to September) and winter (January to March and October to December). Results are shown in Table 6. The following appreciations can be stated:

- Most of the models, with the exception of the naive during summers, do not show a tendency to under- or overestimate, as it can be seen from their low bias values. In the case of the naive model, the underestimation is due to the power forecast being 0 for all nights.

- MAE and RMSE (both absolute error measures) along with Skill (based on RMSE) show an ongoing improvement with the initially proposed models (LSTM better than FF+day input which is in turn better than the first FF and naive models) for summer, winter and whole year altogether. It can also be seen how LSTM and GRU achieve very similar results that outperform the rest of alternatives followed closely by FFbig. SVR is somewhere in between the FF and the FF+day models.

- MAPE shows a somewhat erratic behaviour though, with the GRU model outperforming every other approach, followed by the LSTM model.

- The feedforward model FFbig has a Skill of $51.14 \%$, which is superior to the previous feedforward models, but not to the LSTM model, the architecture of which is proven to be superior for a time-dependent problem like the one at hand. However, this model also proves how feedforward models can achieve better results with bigger architectures and more inputs.

- Unexpectedly, the LSTM model using DNI (LSTMDNI) achieves worse results than those obtained by the original LSTM (46.46\% against 55.03\% skill). This might be related to the results seen in Fig. 6, where the model is seen to overestimate significantly the production, possibly due to a bad DNI forecast from ECMWF. 
Table 6: Performance results for different seasons

\begin{tabular}{|c|c|c|c|c|c|c|c|}
\hline Model & Season & MAE & Bias & MAPE & RMSE & Skill & Correlation \\
\hline \multirow{3}{*}{ Naive } & All & 0.24 & -0.11 & 222.88 & 0.35 & 0 & 0.90 \\
\hline & Summer & 0.32 & -0.27 & 71.05 & 0.40 & 0 & 0.93 \\
\hline & Winter & 0.16 & 0.06 & 419.62 & 0.28 & 0 & 0.87 \\
\hline \multirow{3}{*}{ FeedForward } & All & 0.18 & -0.01 & 296.43 & 0.25 & 27.71 & 0.94 \\
\hline & Summer & 0.21 & -0.06 & 122.66 & 0.28 & 30.74 & 0.94 \\
\hline & Winter & 0.14 & 0.04 & 521.61 & 0.22 & 21.65 & 0.91 \\
\hline \multirow{3}{*}{ FF + day } & All & 0.16 & -0.01 & 209.20 & 0.23 & 34.11 & 0.95 \\
\hline & Summer & 0.20 & -0.02 & 155.72 & 0.26 & 34.53 & 0.94 \\
\hline & Winter & 0.11 & -0.01 & 278.50 & 0.19 & 33.24 & 0.93 \\
\hline \multirow{3}{*}{ LSTM } & All & 0.10 & -0.02 & 90.40 & 0.16 & 55.03 & 0.98 \\
\hline & Summer & 0.12 & -0.02 & 54.29 & 0.17 & 57.02 & 0.98 \\
\hline & Winter & 0.07 & -0.01 & 137.20 & 0.14 & 51.08 & 0.96 \\
\hline \multirow{3}{*}{ SVR } & All & 0.17 & 0.03 & 993.97 & 0.24 & 31.80 & 0.95 \\
\hline & Summer & 0.19 & -0.02 & 178.76 & 0.26 & 35.76 & 0.95 \\
\hline & Winter & 0.15 & 0.08 & 2050.39 & 0.21 & 24.10 & 0.92 \\
\hline \multirow{3}{*}{ FFbig } & All & 0.10 & -0.02 & 91.09 & 0.17 & 51.14 & 0.97 \\
\hline & Summer & 0.13 & -0.03 & 62.06 & 0.19 & 52.53 & 0.97 \\
\hline & Winter & 0.08 & -0.02 & 128.72 & 0.15 & 48.32 & 0.96 \\
\hline \multirow{3}{*}{ GRU } & All & 0.10 & -0.01 & 80.50 & 0.16 & 55.12 & 0.98 \\
\hline & Summer & 0.12 & -0.01 & 64.32 & 0.17 & 56.28 & 0.97 \\
\hline & Winter & 0.07 & -0.02 & 101.46 & 0.13 & 52.74 & 0.97 \\
\hline \multirow{3}{*}{ LSTMDNI } & All & 0.11 & 0.02 & 98.03 & 0.19 & 46.46 & 0.97 \\
\hline & Summer & 0.13 & 0.04 & 76.24 & 0.22 & 46.03 & 0.96 \\
\hline & Winter & 0.08 & -0.01 & 126.28 & 0.15 & 47.38 & 0.96 \\
\hline
\end{tabular}

As previously discussed, MAPE for the different models shows an erratic behaviour. In order to further understand this evolution, new results are computed which include separated values of MAE and MAPE for the different models during day and night (Table 7). It can be seen that the main period for which the naive clearly outperforms other models in terms of MAPE are nights, specially in winter. However, it can be deduced from MAE that absolute errors are similar in both cases and close to 0 (except for the SVR model, which offers here a worst result) but relative values grow because real values (in the denominator of MAPE) are also very small. For this reason, this indicator is disregarded in this work for comparison purposes. Note also how, when splitting nights and days, LSTM and GRU still offer a very similar global performance. However, the GRU model achieves a slightly better correlation for winter days and worse results for summer ones.

\section{Conclusions}

This paper has introduced three deep learning-based forecasting models for the continuous prediction of the aggregated CSP energy production in Spain. They progressively incorporate more inputs and more advanced architectures (that allow the third model to outperform its predecessors). In the same way, the models have been also compared against a naive approach (based on the simple aggregation of the expected irradiance in the locations of the main CSP facilities) and against other models proposed in the literature such as SVR and GRU. All the forecasts provided by the models introduced are updated every 12 hours, when updated NWPs become available.

The performance of the different forecast models has been analysed by computing the most wide range of metrics in the literature for a whole year of CSP energy production. 
Table 7: MAE, MAPE and correlation for summer, winter,day and night

\begin{tabular}{|c|c|c|c|c|c|c|c|c|c|c|c|c|}
\hline \multirow[b]{3}{*}{ Model } & \multicolumn{4}{|c|}{ MAE } & \multicolumn{4}{|c|}{ MAPE } & \multicolumn{4}{|c|}{ Correlation } \\
\hline & \multicolumn{2}{|c|}{ Summer } & \multicolumn{2}{|c|}{ Winter } & \multicolumn{2}{|c|}{ Summer } & \multicolumn{2}{|c|}{ Winter } & \multicolumn{2}{|c|}{ Summer } & \multicolumn{2}{|c|}{ Winter } \\
\hline & Day & Night & Day & Night & Day & Night & Day & Night & Day & Night & Day & Night \\
\hline Naive & 0.29 & 0.37 & 0.27 & & 54.35 & 100 & 660.08 & 100 & 0.92 & - & 0.85 & - \\
\hline FF & 0.22 & 0.19 & 0.21 & 0.08 & & 206.73 & & 792.06 & 0.93 & 0.52 & 0.90 & 0.26 \\
\hline $\mathrm{FF}+$ day & 0.21 & 0.18 & 0.17 & 0.05 & 93.83 & 262.54 & 212.90 & 365.70 & 0.96 & 0.56 & 0.91 & 0.59 \\
\hline LSTM & 0.13 & 0.09 & 0.12 & $\mathbf{0 . 0 3}$ & 33.61 & 90.11 & 85.30 & 206.18 & 0.97 & 0.85 & 0.95 & 0.89 \\
\hline FFbig & 0.14 & 0.09 & 0.13 & 0.03 & 41.59 & 97.53 & 95.73 & 172.58 & 0.96 & 0.84 & 0.95 & 0.88 \\
\hline SVR & 0.24 & 0.10 & 0.18 & 0.12 & 113.02 & 293.07 & 818.09 & 3688.41 & 0.93 & 0.85 & 0.92 & 0.54 \\
\hline GRU & 0.13 & 0.10 & 0.12 & 0.03 & 37.91 & 110.12 & 97.59 & 106.60 & 0.97 & 0.84 & 0.96 & 0.90 \\
\hline LSTMDNI & 0.15 & 0.10 & 0.14 & 0.03 & 45.30 & 129.92 & 102.68 & 157.64 & 0.95 & 0.81 & 0.94 & 0.89 \\
\hline
\end{tabular}

Among the different models proposed, LSTM together with GRU architectures show the superior performance, as expected because of the time-dependent nature of the problem. Among the rest of proposals, the feedforward with a high number of parameters follows in terms of performance. SVR and the initial feedforward models come next, with a better forecast obtained by the SVR model in summer and for the FF+day in winter. Finally, the LSTM with the DNI forecast as input underperforms when compared to the initial LSTM (with TOA irradiance and cloud cover forecast as inputs).

\section{Acknowledgments}

The authors would like to thank the financial support provided by the Universitat Jaume I from Castelló (Spain), the Generalitat Valenciana (GV), and the European Social Fund (ESF). This work was developed within the context of the project with code UJI-B2017-26 and grant number ACIF/2019/106.

\section{References}

[1] Asociación Española para la promoción de la Industria Termosolar, Protermosolar. URL www. protermosolar.com

[2] R.E. de España, Las energías renovables en el sistema eléctrico español 2017 (2018).

[3] J. Hinkley, B. Curtin, J. Hayward, A. Wonhas, R. Boyd, C. Grima, A. Tadros, R. Hall, K. Naicker, A. Mikhail, Concentrating solar powerdrivers and opportunities for cost-competitive electricity, CISRO, Canberra.

[4] R. Sioshansi, P. Denholm, The value of concentrating solar power and thermal energy storage, IEEE Transactions on Sustainable Energy 1 (3) (2010) 173-183. doi:10.1109/TSTE.2010.2052078.

[5] L. Ramírez, J. Vindel, Forecasting and nowcasting of DNI for concentrating solar thermal systems, in: Advances in Concentrating Solar Thermal Research and Technology, Elsevier, 2017, pp. 293-310. doi:10.1016/B978-0-08-100516-3.00013-7.

[6] E. W. Law, A. A. Prasad, M. Kay, R. A. Taylor, Direct normal irradiance forecasting and its application to concentrated solar thermal output forecasting - A review, Solar Energy 108 (2014) 287-307. doi:10.1016/j.solener.2014.07.008.

[7] J. Usaola, Operation of concentrating solar power plants with storage in spot electricity markets, IET Renewable Power Generation 6 (1) (2012) 59. doi:10.1049/iet-rpg.2011.0178.

[8] R. Dominguez, L. Baringo, A. Conejo, Optimal offering strategy for a concentrating solar power plant, Applied Energy 98 (2012) $316-325$. doi:10.1016/j.apenergy.2012.03.043.

[9] G. P. Zhang, M. Qi, Neural network forecasting for seasonal and trend time series, European Journal of Operational Research 160 (2) (2005) 501-514. doi:10.1016/j.ejor.2003.08.037.

[10] S. A. Kalogirou, Applications of artificial neural networks in energy systems, Energy Conversion and Management 40 (10) (1999) $1073-1087$. doi:10.1016/S0306-2619(00)00005-2.

[11] L. Martín, L. F. Zarzalejo, J. Polo, A. Navarro, R. Marchante, M. Cony, Prediction of global solar irradiance based on time series analysis: Application to solar thermal power plants energy production planning, Solar Energy 84 (10) (2010) $1772-1781$. doi:10.1016/j.solener.2010.07.002.

[12] L. Benali, G. Notton, A. Fouilloy, C. Voyant, R. Dizene, Solar radiation forecasting using artificial neural network and random forest methods: Application to normal beam, horizontal diffuse and global components, Renewable Energy 132 (2019) $871-884$. doi:10.1016/j.renene.2018.08.044. 
[13] A. Gensler, J. Henze, B. Sick, N. Raabe, Deep Learning for solar power forecasting - An approach using AutoEncoder and LSTM Neural Networks, in: 2016 IEEE International Conference on Systems, Man, and Cybernetics (SMC), IEEE, 2016, pp. 002858-002865. doi:10.1109/SMC.2016.7844673.

URL http://ieeexplore.ieee.org/document/7844673/

[14] W. Lee, K. Kim, J. Park, J. Kim, Y. Kim, Forecasting Solar Power Using Long-Short Term Memory and Convolutional Neural Networks, IEEE Access 6 (2018) 73068-73080. doi:10.1109/ACCESS.2018.2883330.

URL https: //ieeexplore.ieee.org/document/8543793/

[15] A. A. Prasad, R. A. Taylor, M. Kay, Assessment of solar and wind resource synergy in Australia, Applied Energy 190 (2017) $354-367$. doi:10.1016/j.apenergy.2016.12.135.

URL https://linkinghub.elsevier.com/retrieve/pii/S0306261916319183

[16] C. I. Hussain, B. Norton, A. Duffy, Technological assessment of different solar-biomass systems for hybrid power generation in Europe, Renewable and Sustainable Energy Reviews 68 (2017) 1115-1129. doi:10.1016/j.rser.2016.08.016. URL https://linkinghub.elsevier.com/retrieve/pii/S1364032116304300

[17] K. Nithyanandam, R. Pitchumani, Techno-Economic Analysis of Concentrating Solar Power Plants With Integrated Latent Thermal Storage Systems, in: ASME 2013 7th International Conference on Energy Sustainability, American Society of Mechanical Engineers, 2013. doi:10.1115/ES2013-18213

[18] F. Molteni, R. Buizza, T. N. Palmer, T. Petroliagis, The ECMWF ensemble prediction system: Methodology and validation, Quarterly Journal of the Royal Meteorological Societydoi:10.1256/smsqi.52904.

[19] F. M. Lopes, R. Conceição, H. G. Silva, T. Fasquelle, R. Salgado, P. Canhoto, M. Collares-Pereira, Short-term forecasts of DNI from an integrated forecasting system (ECMWF) for optimized operational strategies of a central receiver system, Energiesdoi:10.3390/en12071368.

[20] J. Dersch, M. Schroedter-Homscheidt, K. Gairaa, N. Hanrieder, T. Landelius, M. Lindskog, S. Müller, L. Ramirez Santigosa, T. Sirch, S. Wilbert, Impact of DNI nowcasting on annual revenues of CSP plants for a time of delivery based feed in tariff, Meteorologische Zeitschriftdoi:10.1127/metz/2019/0925

[21] R.E. de España, Esios. Sistema de información del Operador del Sistema

URL https://www.esios.ree.es/es/analisis

[22] C. Tofallis, A better measure of relative prediction accuracy for model selection and model estimation, Journal of the Operational Research Societydoi:10.1057/jors.2014.103

[23] G. Pfister, R. L. McKenzie, J. B. Liley, A. Thomas, B. W. Forgan, C. N. Long, Cloud Coverage Based on All-Sky Imaging and Its Impact on Surface Solar Irradiance, Journal of Applied Meteorologydoi:10.1175/1520-0450(2003)042;1421:CCBOAI $i 2.0$. CO $_{2} 2$

[24] K. Chhatbar, R. Meyer, The influence of meteorological parameters on the energy yield of solar thermal plants, 2011.

[25] Y. Lecun, Y. Bengio, G. Hinton, Deep learning, Nature 521 (7553) (2015) 436. arXiv:arXiv:1312.6184v5, doi:10.1038/nature14539.

[26] P. Baldi, P. Sadowski, D. Whiteson, Searching for exotic particles in high-energy physics with deep learning, Nature Communications 5 (2014) 4308. arXiv:1402.4735, doi:10.1038/ncomms5308.

[27] C. Olah, Understanding lstm networks.

[28] Y. Bengio, P. Simard, P. Frasconi, Learning Long-Term Dependencies with Gradient Descent is Difficult, IEEE Transactions on Neural Networks 5 (2) (1994) 157-166. arXiv:arXiv:1211.5063v2, doi:10.1109/72.279181.

[29] S. Hochreiter, J. Schmidhuber, Long Short-Term Memory, Neural Computation 9 (8) (1997) 1735-1780. arXiv:1206.2944, doi:10.1162/neco.1997.9.8.1735.

[30] K. Cho, B. van Merrienboer, C. Gulcehre, D. Bahdanau, F. Bougares, H. Schwenk, Y. Bengio, Learning Phrase Representations using RNN Encoder-Decoder for Statistical Machine Translation arXiv:1406.1078. 